\title{
Principle explanation and strategic schema abstraction in problem solving
}

\author{
ALLAN B. I. BERNARDO \\ De La Salle University, Manila, Philippines
}

\begin{abstract}
This study was undertaken to examine the effects of strategic schema-acquisition tasks (problem comparison or problem construction) and the method of principle explanation (abstract or embedded principle method) on schema acquisition. Ninety-eight subjects studied a set of problems in probability, presented according to either method of principle explanation. Half the subjects in each principleexplanation group were then asked to compare analogous problems, and the rest constructed new analogous problems. To determine whether subjects generalized problem schemas, they were given new analogous problems to solve. The results showed that when the abstract principle method was used, schema acquisition was better in problem comparison; but with the embedded principle method, schema acquisition was better in problem construction. Results were discussed in relation to the importance of some fit between the presentation of problem information and the processes that will draw from or build on this information in tasks designed to allow novice problem solvers to acquire advanced problem representations.
\end{abstract}

In many domains of formal learning, such as mathematics, one of the goals of learning and teaching is to develop students' knowledge of the abstract principles and procedures that they use to solve problems in that domain. Research has identified analogical problem solving as one of the means by which students can develop such abstract knowledge for solving problems. In particular, research has shown that through the use of earlier source problems to solve new analogous problems, problem solvers come to develop problem schemas.

Most researchers (for a review, see Reeves \& Weisberg, 1994) agree that abstract principles and procedures can be derived from solving individual analogous problems, and that this abstract information can be represented independently of the analogous problems themselves. Moreover, research in analogical problem solving suggests that a wide range of problem-specific factors such as the content domain of the problem, superficial or sur-

This research was supported by a Spencer Postdoctoral Research Fellowship awarded to the author by the National Academy of Education, United States of America. The paper was completed while the author was a Visiting Scholar at the Faculty of Psychology and Educational Sciences, Leuven University, Belgium, and with partial support from the College of Education Research Fund, De La Salle University, Manila. I thank the students, teachers, and administrators of Lourdes School and St. Mary's College, both in Quezon City, for their enthusiastic participation in the study. I also acknowledge the valuable assistance provided by Niño Mateo, Alma San Buenaventura, Chinee Tan, Annalyn de Guzman, Raffy Banaag, Jezzle Ticao, Sylvia Sajo, Christopher Cadua, and Sendy Mangilit in preparing the research materials and gathering the experimental data. Finally, I thank two anonymous reviewers for their most thoughtful comments on earlier versions of this paper. Correspondence should be addressed to the author at the College of Education, De La Salle University, 2401 Taft Avenue, Manila, 1004 Philippines (e-mail: claabb@dlsu.edu.ph). face elements of the problem, and the problem-solving context play an important role in the development of schemas. For example, the reminding model of Ross (1987, 1989; Medin \& Ross, 1989) and the multiple-trace model of Hintzman (1986) assume that content information plays very important roles in driving the retrieval and mapping processes that are integral to analogical problem solving, and that as such, content information acquires functional significance in the problem-solving processes.

These models are generally well supported by empirical evidence (see Reeves \& Weisberg, 1994, for a detailed review). Numerous studies have documented the effects of problem content on the retrieval of relevant source problems for analogical problem solving (Blessing \& Ross, 1996; Novick \& Holyoak, 1991; Reed, Ackinclose, \& Voss, 1990; Ross, 1989), although content may affect some domains more than others (Bassok \& Holyoak, 1989). Studies also show that content affects the complex process of mapping between the source and target analogous problems (Bassok, 1990; Bassok, Chase, \& Martin, 1998; Novick \& Holyoak, 1991; Ross, 1987, 1989) even with highly experienced (Blessing \& Ross, 1996) and expert problem solvers (Novick, 1988). Similar effects on retrieval and mapping of analogous information have been found for factors other than the immediate content elements of the problem (Bernardo, 1998; Spencer \& Weisberg, 1986). These findings suggest that aspects of the content of the problem and the problem-solving episode must be retained in the memory representations developed after analogical problem solving. Concrete problemspecific information is retained because it is assumed to be useful for guiding the retrieval and use of abstract information (Bernardo, 1994; Medin \& Ross, 1989; Ross \& Kennedy, 1990). 
The present experiment was carried out in an attempt to investigate further the effects of more concrete aspects of the problem-solving episode by looking at two factors: the manner in which abstract solution principles are presented, and the learning task given to facilitate the strategic abstraction of the schema.

The abstract principles used to solve problems can be presented in different ways; Ross and Kilbane (1997) have distinguished two. The first, called the abstract principle method, involves the approach typically used in instruction in mathematics. A general principle is given and explained, and then a problem is given to illustrate how the principle is applied. The second is the embedded principle method, whereby a problem illustrating a specific principle is given and the principles for solving the problem are integrated with the completion of the problem solution. Hence, there is no explicit presentation of the principles that one uses to solve the problem. Ross and Kilbane studied how these two modes of presenting principles affected the processes of analogical problem solving. They found that problem solvers were likely to be misled by non-analogous superficial similarities when the abstract principles were presented explicitly. They concluded that with this method, the problem solver relies heavily on the features of the source problem but that these features are not yet meaningfully linked to the abstract solution information. Thus, the problem solver becomes more vulnerable to misleading superficial similarities between the source problem and the analogous problems or among analagous problems. But with the embedded principle method, the problem solver seems to acquire more contextualized knowledge about the problem solutions. When solving new analogous problems, the problem solver is less susceptible to misleading superficial similarities, because the abstract principles that need to be applied are functionally related to other elements of the episodic memory trace for the source problem.

If there are at least two ways in which abstract solution principles may be presented, there are several ways by which problem solvers can be made to encode the structural features of a problem to facilitate problem schema acquisition. These strategic schema acquisition tasks assume that problems solvers must intentionally encode, use, and/or apply the structural elements of the analogous problems in order for schema acquisition to take place (Reeves \& Weisberg, 1994). One of the most often studied tasks is analogical problem comparison. For example, Catrambone and Holyoak (1989) found that subjects who were asked to synopsize the similarities of two analogous problems achieved higher rates of transfer in comparison with those who just summarized the two problems. Cummins (1992) found that asking subjects to compare two analogous problems in terms of their solutionrelevant information led to better abstraction of problem category information than did asking them to compare the problems in terms of content information. Analogical problem comparison is assumed to be effective for schema acquisition because it forces the problem solver to sort the relevant structural information from the irrelevant surface information, thus facilitating the development of abstract problem schemas.

Recently, another task that requires the problem solver to focus on structural information has been studied. Bernardo (2001) studied the effects of analogical problem construction on analogical transfer. This task involves asking subjects to construct their own problems after they have been presented exemplars of a problem category. A series of experiments showed that this task was effective in facilitating acquisition of schema information and transfer. The task was assumed to involve the reverse of the mapping process that is essential to analogical problem solving. In analogical problem solving, the problem solver is required to map out the similarities between the analogous source and target problems, and this mapping leads to the abstraction of a general principle (Novick \& Holyoak, 1991; Ross \& Kennedy, 1990). In analogical problem construction, the problem solver creates a new problem by projecting new problem elements that would map with the elements of the original problem.

Studying the mode of principle explanation and the type of schema-acquisition task has both theoretical and practical significance. Both of these variables relate to instructional processes for teaching problem solving in formal schools. Ross and Kilbane (1997) have suggested that looking at such variables will not only contribute to our understanding of the basic cognitive processes that occur in analogical problem solving and schema acquisition, but also provide some practical insights into how instructional processes can be changed to facilitate better learning of abstract problem information.

It was not the objective of the study presented here to ascertain which mode of principle explanation or strategic schema acquisition task might be better. Indeed, the notion that various modes of schema acquisition may all lead to the abstraction and generalization of structural problem information has been suggested by a number of researchers (e.g., Reeves \& Weisberg, 1994; Ross \& Kennedy, 1990). The objective was rather to find out whether the effectiveness of strategic schema acquisition tasks would be affected by how the abstract solution information and the exemplar problems were presented to the problem solvers.

\section{EXPERIMENTAL DESIGN}

There were two phases in the experiment: the study phase and the transfer test phase. Two independent variables, the method of principle explanation and the strategic schema acquisition task, were manipulated in the study phase. The subjects were asked to study a set of problems with a description of the underlying principles and a worked-out solution. The principles were presented using either the abstract principle method or the embedded principle method. After studying each problem, the subjects were required to either construct their own analogous problem or compare two analogous problems. Thus, there were four conditions with different combinations of two methods of principle explanation and two analogical tasks. 
The study phase was followed immediately by the transfer test phase. All the subjects were given a new set of analogous word problems, and they were asked to solve the problems without directly referring to the previous problems. The subjects' performance on these problems was scored for transfer. Successful transfer was the indirect measure of schema acquisition used in the experiment. This indirect measure was used because numerous studies have shown that the effective abstraction of schema information from source problems strongly predicts transfer to analogous problems (Catrambone \& Holyoak, 1989; Chi, Bassok, Lewis, Reimann, \& Glaser, 1989; Novick \& Holyoak, 1991; Spencer \& Weisberg, 1986).

\section{METHOD}

\section{Subjects}

The subjects were 98 male and female 3rd-year high school students in two private high schools in Metro Manila who participated as part of a class activity. All the students had completed 2 years of high school algebra, and had not taken any course on probability. Fifty subjects were randomly assigned to the embedded principle condition, and 48 to the abstract principle condition. For each condition, half the subjects were randomly assigned to do analogical problem construction and half were asked to do analogical problem comparison.

\section{Materials}

Four types of problems in basic probability were used: conjunction problems with independent events, conjunction problems with dependent events, disjunction problems with exclusive events, and disjunction problems with intersecting events. The first three problem types involved either adding or multiplying the individual probabilities of two events. The last problem type involved adding and subtracting probabilities corresponding to the likelihood of two events. These types of problems were chosen because the principles involved were all unfamiliar to the subjects, but the subjects' background knowledge in algebra could allow them to work through the problem solutions. (Appendix A lists examples for each of the problem types.)

For each problem type, 4 analogous problems were created, making a total of 16 problems. Each analogue involved a superficially different set of objects, relations, and stories. For each problem, a study sheet was made, following one of two approaches. Study sheets using the abstract principle method started with an abstract description of the principles underlying the problem solution. This description was then followed by an example of a problem and the instantiation of the solution procedures. Study sheets using the embedded principle method started with a problem followed by the solution procedures; the principles were not explicitly stated but were integrated within the description of the solution procedures. (See Appendices B and C for examples of study sheets following the two methods.)

For the analogical problem construction condition, the study sheet was attached to another sheet, which was used for the construction task. In this attached sheet, the subject was told to make his/her own problem similar to the one just studied. The subjects were also given suggestions regarding objects and events they could use in the problems they would create. For the analogical problem comparison condition, the study sheet was also attached to another sheet. This study sheet included a new analogous word problem, for which they were asked to compare the similarities between the new word problem and the one just studied.

From the 16 problems, four clusters of problems were created; each of the 4 problems within each cluster came from a different one of the four problem types. The study sheets for each cluster were arranged so that the two conjunction problems were always paired in sequence and the two disjunction problems were also paired in sequence. The cluster of problems that each subject worked on was assigned randomly. The sequence of problems within a pair and the sequence of pairs were counterbalanced across subjects.

Transfer test sheets were prepared for each of the 16 problems. In the transfer test sheets, the problem was typed on top of the page and the rest of the page was left blank for the solutions. The test sheets were combined in the same four clusters as were the study sheets, but the sheets were arranged in random order. A subject information sheet was also made, on which the subjects were asked for basic personal information, including details about their backgrounds in mathematics and languages.

All the materials for each subject were combined in one booklet which contained (1) the general instructions for the task and specific instructions for the study phase, (2) the four study sheets for one cluster, which were attached to the analogical problem construction or comparison sheets, (3) the instruction for the test phase, (4) the four test sheets from another cluster, and (5) the subject information sheet.

\section{Procedure}

The subjects participated in groups of 10 to 15 . They were asked to sign an informed consent form and were then given a test booklet appropriate to their randomly assigned condition. They were asked to read the instructions carefully, and the experimenter answered any questions that they had about the task. After these questions had been answered, the subjects proceeded to the study phase. They were given 2 min to study each problem, and they were informed when they had $30 \mathrm{sec}$ left. For the analogical problem construction condition, they were instructed to create their own analogous problem after each study; in particular, they were told to create a problem of their own that could be solved using the solution procedure that they had just studied. They were told that they should try to solve the problems that they had created. For the analogical problem comparison conditions, they were instructed to compare the given problem with the one they had just studied. They were also told that they should try to solve the new problem. The subjects were given 4 min to accomplish both the analogical problem construction and comparison tasks, and they were told when they had $30 \mathrm{sec}$ left.

After the subjects had completed the study phase, they were asked to read the instructions for the test phase. The experimenter answered all questions about this part of the study, and the subjects proceeded to the test problems. They were given $3 \mathrm{~min}$ to answer each test problem without directly referring to the study problems; they were also told when they had $30 \mathrm{sec}$ left. For both the study and test phases, the subjects were told that they could not proceed to the next problem if they finished ahead of time, nor could they go back to study or to work on any previous problem. After the subjects had completed the four test problems, they answered the subject information sheet and they were informed about the basic purpose of the study. The experimenter then answered any questions that they had about the study.

\section{RESULTS}

The subjects' answers for the test phase were coded for transfer. The solutions were coded as showing complete transfer (one point), transfer-with-error (two-thirds point), partial transfer (one-third point), or no transfer (zero). Complete transfer was scored if the subject arrived at the correct final answer by using the solution given in the study phase. Transfer-with-error was scored if the subject used the same equation and procedures as in the study phase, but made some error in computation. 
Table 1

Mean Transfer Scores as a Function of Principle Explanation and Schema Acquisition Task

\begin{tabular}{lccccc}
\hline \multirow{2}{*}{ Method } & \multicolumn{2}{c}{ Problem Comparison } & & \multicolumn{2}{c}{ Problem Construction } \\
\cline { 2 - 3 } \cline { 5 - 6 } & $M$ & $S E$ & & $M$ & $S E$ \\
\hline Abstract principle & 0.312 & 0.048 & & 0.194 & 0.045 \\
Embedded principle & 0.176 & 0.147 & & 0.303 & 0.051 \\
\hline
\end{tabular}

Partial transfer was scored if the subject used the correct solution equation, but was not able to map or apply it correctly for the new problem. All other solutions were scored as no transfer. For each subject, the average transfer score across the four problem types were computed and analyzed in a two-way analysis of variance, with principle explanation and schema acquisition task as betweengroups factors. The mean transfer score for the different study conditions are summarized in Table 1 .

The results of the analysis showed no statistically reliable main effect for either factor (both $F \mathrm{~s}<1.0$ ). However, there was a reliable interaction between principle explanation and schema acquisition task $[F(1,94)=7.65$, $\left.M S_{\mathrm{e}}=0.05, p<.007\right]$. To explore the nature of the interaction, the scores were analyzed for simple effects. The simple effect of schema acquisition task on the embedded principle condition was statistically reliable $[F(1,48)=$ $\left.4.59, M S_{\mathrm{e}}=0.04, p<.04\right]$. Subjects who completed analogical problem construction attained higher transfer scores than did those who completed analogical problem comparison. There was a marginally reliable simple effect of schema acquisition task on the abstract principle condition $\left[F(1,46)=3.20, M S_{\mathrm{e}}=0.05, p=.08\right]$. Subjects who completed analogical problem comparison attained higher transfer scores than did those who completed analogical problem construction; this result is the opposite of that for the embedded principle condition.

\section{DISCUSSION}

The study was undertaken to study the effects of two modes of principle explanation and two strategic schema acquisition tasks on the abstraction of problem schemas. The results do not indicate the advantage of using one mode of principle explanation over the other. Neither was there any advantage of using one schema acquisition task as opposed to the other. The benefit of using a specific mode of principle explanation seems to depend on the nature of the schema acquisition task. The general pattern of results seems consistent with the assertion that various modes of schema acquisition may lead to the abstraction of structural problem information (Reeves \& Weisberg, 1994; Ross \& Kennedy, 1990). Yet the results are also somewhat surprising. Previous research (Ross \& Kilbane, 1997) has found a clear advantage of using the embedded principle method as opposed to the abstract principle method. The results of the present experiment suggest that this might not always be the case. A couple of hypotheses can be posed to account for this pattern of results.
The first hypothesis can be referred to as a matching hypothesis. Schema acquisition is most effective when there is a match between the type of information provided about the principles and the type of information needed in the schema abstraction task. In particular, the abstract, decontextualized information provided in the abstract principle method would be more suited for the analogical problem comparison task, because the abstract information could be used to effectively guide that comparison of the important structural features of the analogous problems. Such abstract information would not be as useful in the analogical problem construction task, because the task requires more contextualized information. In this regard, the embedded information from the other method seems more suitable, because there are more meaningful connections between the abstract and concrete features of the problem.

A second hypothesis can be referred to as the information completion hypothesis. ${ }^{1}$ The effective combination of principle explanation and schema abstraction might be one in which the limitations of the presented information are complemented by the schema acquisition task. This hypothesis is based on the assumption that effective transfer requires both abstract principles and a means of linking this abstract information to concrete problems. Thus, abstract principle information might be more effective with analogical problem comparison, because the comparison provides the problem solver with two sets of concrete problem information within which to embed and contextualize the abstract problem information. On the other hand, the embedded principle information might be more effective with analogical problem construction, because the latter requires the problem solver to abstract the structural features of the problem, which will be the basis of features of the analogous problem being constructed. This requirement prompts the problem solver to extract the abstract information embedded within the given problem information. In other words, the schema acquisition task that best allows for the completion of the information needed for analogical transfer will be the most effective one, but all that depends on what information is already available and what is not.

What the results suggest is that learning tasks intended to allow novice problem solvers to acquire more advanced problem schemas should be designed in ways that take into account how the problem-solving principles are presented. The two hypotheses stated above indicate two possible ways in which the method of presenting principles could influence the effectiveness of strategic schema acquisition tasks, or, put another way, how different strategic schema acquisition tasks could best involve the use of different forms of problem information. These hypotheses (and other unexplored ones) can be studied further as we come to know more about the specific nature of the differences between the two modes of principle explanation and between the two schema acquisition tasks.

One way of testing these two hypotheses would be to involve some test of whether the problem schemas formed 
by subjects in the abstract principle and analogical problem comparison include only (or mainly) abstract information or a combination of abstract information with concrete contextual information. If the matching hypothesis were found to be correct, we could assume that the schemas formed should be mainly abstract information and that the successful transfer of information to solve a new analogical problem would be driven mainly by the operation of abstract problem information. On the other hand, if the information completion hypothesis were found to be correct, we could assume that the schemas formed would include both abstract and concrete information, and that the successful analogical problem solution was possible because the concrete information enabled the application of the abstract information.

Even as the specific processes that shaped the results of the present study are not yet certain, we can already draw some important insights from the findings. Instructional variables were chosen as independent variables in the experiment because they were deemed useful for revealing properties of the analogical transfer and schema acquisition processes. Consistent with the general findings of Ross and Kilbane (1997), the results of the present study indicate that the manner in which problem information is presented affects analogical transfer and the generalization of problem information. However, the present results also extend Ross and Kilbane's findings by showing that different analogical learning tasks may effectively draw from or build on the information provided by the different forms of principle explanation. Whichever of the two proposed hypotheses turns out to be correct, the present results implicate some principle of transfer appropriate processing. Although the specific meaning of "appropriate" is still in question, the effectiveness of modes of principle explanation and strategic schema-acquisition tasks seems to depend on whether the information provided in the former is appropriate to the processes underlying the latter, or vice versa.

Just as important, the results of the present study point to some consequences of using some instructional tasks instead of others. The main results indicate that the different strategic schema acquisition tasks that can be used will be effective if they somehow match or complement the information that students have formed about the abstract and concrete elements of the relevant problem exemplars. Thus teachers need to be more mindful about how abstract and concrete information about problem types and categories are introduced and explained, because these could constrain later processing of similar, related problem information. The results again qualify the conclusions of Ross and Kilbane (1997) about the effects of modes of principle explanation in problem-solving instruction. Conventional teaching methods in school mathematics tend to follow the abstract principle method, and Ross and Kilbane suggested that such an approach may be limited because it does not provide students with a context within which to develop knowledge about the applicability of the abstract principles. The present results suggest that combining this conventional method of principle explanation with another conventional learning task of requiring students to compare analogical problems can be effective in allowing students to develop advanced problem schemas that enable them to solve new analogous problems. In the present instance, the students did not seem to have too much difficulty in applying the abstract information that they knew.

Simple and straightforward prescriptions about whether one way of presenting information or one way of designing learning tasks may not be appropriate at this point, as the present results suggest. If any prescription can be given to teachers, it is that learning tasks may be tailored to fit the information that students may have already acquired, or that information may be configured in ways that will suit the learning processes to be engaged. The present challenge is to understand how an ideal convergence can be defined and achieved so that students may come to more effectively develop advanced representations of the knowledge that they require in order to be able to solve problems in the various domains of learning in school.

\section{REFERENCES}

Bassok, M. (1990). Transfer of domain-specific problem-solving procedures. Journal of Experimental Psychology: Learning, Memory, \& Cognition, 16, 522-533.

Basso K, M., Chase, V. M., \& Martin, S. A. (1998). Adding apples and oranges: Alignment of semantic and formal knowledge. Cognitive Psychology, 35, 99-134.

BASSOK, M., \& HOLYOAK, K. J. (1989). Information transfer between isomorphic topics in algebra and physics. Journal of Experimental Psychology: Learning, Memory, \& Cognition, 15, 153-166.

Bernardo, A. B. I. (1994). Problem-specific information and the development of problem-type schemata. Journal of Experimental Psychology: Learning, Memory, \& Cognition, 20, 379-395.

Bernardo, A. B. I. (1998). Language format and analogical transfer among bilingual problem solvers in the Philippines. International Journal of Psychology, 33, 33-44.

Bernardo, A. B. I. (2001). Promoting analogical transfer in math problem solving using analogical problem construction. EducationalPsychology, 21, 137-150.

Blessing, S. B., \& Ross, B. H. (1996). Content effects in problem categorization and problem solving. Journal of Experimental Psychology: Learning, Memory, \& Cognition, 22, 792-810.

Catrambone, R, \& Holyoak, K. J. (1989). Overcoming contextual limitations on problem-solving transfer. Journal of Experimental Psychology: Learning, Memory, \& Cognition, 15, 1147-1156.

Chi, M. T., Bassok, M., Lewis, M. W., Reimann, P., \& Glaser, R. (1989). Self-explanations: How students study and use examples in learning to solve problems. Cognitive Psychology, 14, 145-182.

Cummins, D. D. (1992). Role of analogical reasoning in induction of problem categories. Journal of Experimental Psychology: Learning, Memory, \& Cognition, 18, 1103-1124.

HintzMan, D. L. (1986). "Schema abstraction" in a multiple-trace memory model. Psychological Review, 93, 411-428.

Medin, D. L., \& Ross, B. H. (1989). The specific character of abstract thought: Categorization, problem solving, and induction. In R. Sternberg (Ed.), Advances in the psychology of intelligence (Vol. 5, pp. 189-223). Hillsdale, NJ: Erlbaum.

Novick, L. R. (1988). Analogical transfer, problem similarity, and expertise. Journal of Experimental Psychology: Learning, Memory, \& Cognition, 14, 510-520. 
Novick, L. R, \& Holyoak, K. J. (1991). Mathematical problem solving by analogy. Journal of Experimental Psychology: Learning, Memory, \& Cognition, 17, 398-415.

Reed, S. K., Ackinclose, C. C., \& Voss, A. A. (1990). Selecting analogous problems: Similarity versus inclusiveness. Memory \& Cognition, 18, 83-98.

Reeves, L. M., \& WeISBERG, R W. (1994). The role of content and abstract information in analogical transfer. Psychological Bulletin, 115, 381-400.

Ross, B. H. (1987). This is like that: The use of earlier problems and the separation of similarity effects. Journal of Experimental Psychology: Learning, Memory, \& Cognition, 13, 629-639.

Ross, B. H. (1989). Distinguishing types of superficial similarities: Different effects on the access and the use of earlier problems. Journal of Experimental Psychology: Learning, Memory, \& Cognition, 15, 458-468.
Ross, B. H., \& Kennedy, P. T. (1990). Generalizing from the use of earlier examples in problem solving. Journal of Experimental Psychology: Learning, Memory, \& Cognition, 16, 42-55.

Ross, B. H., \& Kilbane, M. C. (1997). Effects of principle explanation and superficial similarity on analogical mapping in problem solving. Journal of Experimental Psychology: Learning, Memory, \& Cognition, 23, 427-440.

SPEncer, R. M., \& Weisberg, R. W. (1986). Context-dependenteffects on analogical transfer. Memory \& Cognition, 14, 442-449.

\section{NOTE}

1. I thank the anonymous reviewers of this manuscript for suggesting arguments for the second hypothesis.

\section{APPENDIX A \\ Examples of Probability Problems Used in the Study}

\section{Conjunction Problem With Independent Events}

There are 76 books in the Science section of the library, 6 of which are new. In the History section, there are 120 books, 15 of which are new. The principal randomly picks a book from each of the two sections. What is the probability that the principal picks a new book from both sections?

\section{Conjunction With Dependent Events}

A total of 40 votes was cast for class president. Dennis received 30 votes and Claudia got the remaining 10 votes. The ballots were tallied one at a time in random order. What is the probability that the first ballot tallied was a vote for Dennis and the second ballot was for Claudia?

\section{Disjunction With Exclusive Events}

There are 150 athletes participating in the intramural sportsfest. Sixty athletes are competing in basketball, 60 in volleyball, and 30 in taekwondo. If an athlete is chosen randomly, what is the probability that the athlete is competing either in basketball or taekwondo?

\section{Disjunction With Intersecting Events}

There are 54 councilors in one city; 34 are with the LAKAS party and the rest are with LABAN. 40 of the councilors, 24 with LAKAS and 16 with LABAN, are lawyers. If a councilor is chosen randomly, what is the probability that the councilor is either with LAKAS or is a lawyer?

\section{APPENDIX B \\ Example of Study Problem Using Embedded Principle Method}

Consider the following example of a CONJUNCTION PROBLEM WITH INDEPENDENT EVENTS:

In Section A, there are 16 female students out of the total of 38 students. In Section B, there are 28 females out of the 42 students. In each section, a student is randomly chosen to be attendance monitor. What is the probability that a female student is chosen attendance monitor in both sections?

To solve the following problem, you first need to answer several questions.

First you need to think about the probability that a female student is randomly chosen as monitor in Section A. How many students are there to choose from in Section A? Out of the possible choices available, how many are female? ____. The probability a female student is chosen in Section A is the ratio of female students to the total number of students (or the number of female students divided by the number of possible choices). This ratio is ____. This quantity is $\mathrm{P}(\mathrm{A})$ or the probability that a female student is randomly chosen monitor in Section A.

Now you need to think about the probability that a female student is randomly chosen as monitor in Section B. How many students are there to choose from in Section B? . Out of the possible choices available, how many are female? _____. The probability a female student is chosen in Section B is the ratio of female students to the total number of students and this ratio is that a female student is randomly chosen monitor in Section B. This quantity is $\mathrm{P}(\mathrm{B})$ or the probability

Now that you know the probability that a female student is chosen in Section A and that in Section B, you can determine the probability that both events occur by multiplying the two independent probabilities. So you can use the equation, $P(A$ and $B)=P(A) \times P(B)$ and the probability that a female student is randomly chosen monitor in both sections is

If your answer is about 0.28 or $28 \%$, you are correct. 


\section{APPENDIX C \\ Example of Study Problem Using Abstract Principle Method}

Suppose that there are two unrelated events, either of which may or may not happen. You want to know the probability that these two separate events will both happen? The problem you have is called a CONJUNCTION PROBLEM WITH INDEPENDENT EVENTS. What will you do to find out the answer?

First you will need to find out two things: (1) the probability that the first event will happen independently, and (2) the probability that the second event will happen independently.

To determine the probability that an event will happen, you will need to consider the ratio of the instances of that event to the total number of instances in that set. That ratio is the probability that the event will happen, and can be represented and computed as

$$
P(\text { Event })=\frac{\# \text { of instances of event }}{\text { total \# of instances in set }}(\# \text { of instances of event } \div \text { total \# of instances })
$$

After you determine the probability of the two independent events, you can determine the probability that both events will happen by multiplying the probabilities of each event. So if the two events are represented by $X$ and $Y$, the probability that both will happened can be computed using the formula: $P(X$ and $Y)=P(X) \times P(Y)$

Consider the following example:

In Section A, there are 16 female students out of the total of 38 students. In Section B, there are 28 females out of the 42 students. In each section, a student is randomly chosen to be attendance monitor. What is the probability that a female student is chosen attendance monitor in both sections?

Think of the probability that a female student is randomly picked as monitor in Section A. There are $16 \mathrm{fe}-$ males in the section out of the total 38 . So the probability is . This is your $\mathrm{P}(\mathrm{X})$.

Now think of the probability that a female student is randomly picked as monitor in Section B. There are 28 females out of the total 42 students. So the probability is This is your $\mathrm{P}(\mathrm{Y})$.

Now that you know the probability of each independent event, you can now determine the probability that both will happen by multiplying the two probabilities. So using the equation, $P(X$ and $Y)=P(X) \times P(Y)$, you now know that the probability that a female student will be randomly chosen attendance monitor in both sections is

If your answer is around 0.28 or $28 \%$, you are correct. 